\title{
Desenvolvimento de um sistema de recomendação de grupos e recursos para apoio à abordagem de temas transversais
}

\author{
Douglas Kellermann - MPIE/IFRS - douglaskellermann@gmail.com \\ Marcelo Augusto Rauh Schmitt - MPIE/IFRS - marcelo.schmitt@poa.ifrs.edu.br \\ Márcia Amaral Corrêa de Moraes - MPIE/IFRS - marcia.correa@ sertao.ifrs.edu.br
}

\begin{abstract}
Resumo. Este artigo apresenta um sistema de recomendação para apoiar a abordagem de temas transversais em cursos superiores. A premissa de que sistemas como este podem contribuir para o processo de ensino e de aprendizagem embasa o desenvolvimento de um plugin para o ambiente Moodle. $O$ software tem como objetivo facilitar a prática da transdisciplinaridade através do agrupamento de estudantes e da recomendação de materiais de aprendizagem. Os testes simulados indicam uma ferramenta relevante e útil para a abordagem de diferentes temas transversais no ensino superior.
\end{abstract}

Palavra-chave: Sistema de recomendação educacional, Ambiente virtual de aprendizagem, Transdisciplinaridade.

\section{Development of a group and resource recommendation system to support cross-cutting theme teaching}

\begin{abstract}
This article presents a recommendation system to support cross-cutting approaches in higher education. The premise that systems like this can contribute to the teaching and learning process is the development of a plugin for the Moodle environment. software aims to facilitate the practice of transdisciplinarity through the grouping of students and the recommendation of learning materials. The simulated tests indicate a relevant and useful tool to approach different cross-cutting themes in higher education.
\end{abstract}

Keywords: Educational recommendation system, Virtual learning environment, Transdisciplinarity.

\section{Introdução}

Pesquisadores do campo da educação que se debruçam sobre a questão da transdisciplinaridade (BUSQUETS et al., 2003; ARAÚJO, 2014) argumentam que temas transversais deveriam ser umas das questões centrais do currículo e que os conteúdos disciplinares deveriam ser um meio para a reflexão de problemas sociais. No contexto do ensino superior, percebe-se alinhamento neste sentido, através do Parecer CNE/CES $n^{0}$ 776/1997. Tal documento determina que, em sua elaboração, as diretrizes curriculares para os cursos do ensino superior devem:

contemplar elementos de fundamentação essencial em cada área do conhecimento, campo do saber ou profissão, [...] Devem induzir a implementação de programas de iniciação científica [...]. Finalmente, devem incluir dimensões éticas e humanísticas, desenvolvendo no aluno atitudes e valores orientados para a cidadania. (BRASIL, 1997, p. 2) 
Associado a isto, no Brasil, os requisitos legais e normativos, descritos no instrumento de avaliação de cursos de graduação presenciais e a distância (BRASIL, 2015), determinam a inclusão das temáticas das políticas de educação ambiental, de educação em direitos humanos e de educação das relações étnico-raciais nos componentes curriculares. No cumprimento desta determinação, é facultado às Instituições de Ensino Superior (IES) a efetivação destas políticas através de práticas disciplinares, interdisciplinares, transdisciplinares ou mistas.

Observa-se que a alternativa da transdisciplinaridade é menos explorada, perdendo-se oportunidade de atuar para redução da fragmentação disciplinar e buscar melhor instrumentalização dos futuros profissionais e cidadãos para os complexos problemas de nossa sociedade. Por certo, podem haver dificuldades e problemas em operacionalizar tal prática.

A partir deste cenário, este artigo relata o desenvolvimento de um sistema de recomendação que auxilia na formação de grupos para atividades relacionadas aos temas dos requisitos legais com base em conhecimentos prévios e perfis de utilização do ambiente virtual de aprendizagem (AVA). Além disso, o sistema recomenda recursos selecionados para atividades, priorizando os de maior relevância e classificação, privilegiando ainda a qualificação realizada por alunos com mesmo perfil.

O presente trabalho preconiza a coleta de dados implícita no transcorrer do curso considerando as diferentes disciplinas cursadas pelo aluno. $\mathrm{O}$ desenvolvimento do sistema encontra-se finalizado e resultados das simulações realizadas demonstram a conformidade do processamento computacional. Considerados os aspectos positivos de grupos de estudantes adequadamente formados e a utilização de recursos apropriados para realização das atividades colaborativas, entende-se que o sistema representa importante contribuição à prática da transdisciplinaridade.

\section{Transdisciplinaridade no ensino superior}

Mesmo que o desenvolvimento do software aqui relatado atenha-se aos requisitos legais, a flexibilidade da configuração do sistema permite que outros temas transversais possam ser utilizados. A proposta é que a ferramenta possa auxiliar não apenas para o cumprimento desta obrigação legal, mas também para favorecer a prática transdisciplinar de forma mais ampla.

\subsection{Temas transversais e a articulação com conteúdo de componentes curriculares}

Antes de avançar, cabe conceituar interdisciplinaridade como; "coordenação, cooperação e integração entre disciplinas, suas especificidades e seus domínios linguísticos, [...] que demanda diálogo, abertura e atitude colaborativa dos sujeitos no ato de investigar e conhecer juntos" (SUANNO, 2015, p. 109), e transdisciplinaridade; "como o prefixo 'trans' indica, diz respeito àquilo que está ao mesmo tempo entre as disciplinas, através das diferentes disciplinas e além de qualquer disciplina [...]" (NICOLESCU, 1999, p. 50, grifos do autor). A interdisciplinaridade é importante instrumento para transdisciplinaridade na abordagem e articulação de temas transversais.

Considerando que transdisciplinaridade é prática em permanente discussão, cabe esclarecer que quando está-se tratando de abordar temas transversais em conteúdos disciplinares, está-se referindo à inclusão de temática transversal nas disciplinas. Para 
designar o desenvolvimento da temática junto ao conteúdo curricular, usar-se-á a expressão articular, no sentido de ligar ou religar conteúdos ou conhecimentos.

Esta articulação, diante de currículos tradicionais, organizados por disciplinas, amplamente utilizados nas IES, considera que a temática transversal "deve estar incorporada nas próprias disciplinas - Subentende a perspectiva de indissociação da ciência (disciplina) e da formação geral, incluindo temas gerais, educação para cidadania, abrangendo a educação em valores" (ARAÚJO, 2014, p. 32).

Abordar determinados temas em diferentes disciplinas é considerada uma ação desafiadora. A dificuldade pode ser tamanha que torna-se comum resolver a questão lançando mão de disciplinas específicas no currículo para abordar este ou aquele tema.

\subsection{Abordagem de temas transversais na prática}

A inclusão de temas nos conteúdos curriculares pode, então, ocorrer de diferentes formas: disciplinas específicas, abordagens interdisciplinares ou transdisciplinares. Associado a isto, a articulação de temas e conhecimentos em boa parte das disciplinas, por vezes, extrapola a formação e a experiência dos professores. Eventualmente, estas situações fazem surgir argumentos contra transversalidade. Um argumento pró-disciplina específica é que "a transversalidade não funciona na prática, nem há garantias de que ela seja praticada nas escolas e instituições de ensino” (BERNARDES; PRIETO, 2010, p. 178).

A interdisciplinaridade, prática importante na articulação de temas e conteúdos disciplinares, traz à tona exemplos de desafios e apresenta suas próprias dificuldades. Em revisão bibliográfica sobre a interdisciplinaridade no Ensino Médio (MOZENA; OSTERMANN, 2014, p. 199) percebe-se que os principais problemas e dificuldades de implementação da interdisciplinaridade são de ordem institucional (ex. "falta de formação universitária inicial ou continuada para o professor voltada para o trabalho interdisciplinar"), metodológica (ex. "falta de orientação para criação de relações pertinentes entre as disciplinas"), relativas aos professores (ex. "falta de domínio de conteúdos de outras disciplinas") e relativos aos alunos (ex. "assim como os professores, os alunos não consideram a prática legítima”).

Em situações motivadas também em atenção aos requisitos legais, documentos institucionais são inspecionados para quantificar o nível e forma do atendimento à legislação. Dinardi e Araujo (2017) apontam um posicionamento afinado dos coordenadores de curso quanto à importância da abordagem dos temas relacionados à Educação Ambiental. Entretanto, em suas conclusões, é realçada a importância de aprofundar os estudos sobre a qualidade da abordagem praticada em diferentes cursos.

Como pode-se perceber, a prática da transdisciplinaridade é, muitas vezes, desafiadora. De fato, esta é uma importante justificativa para o desenvolvimento do software: contribuir para atuação docente na prática transdisciplinar através de sistema de recomendação educacional.

\section{Sistemas de recomendação educacionais}

Existem diferentes pesquisas sobre sistemas de recomendação para educação. Trabalhos que compartilham o mapeamento destas pesquisas (CAMPOS et al., 2018; AGUIAR et al., 2014) demonstram que são diversos os enfoques das investigações nesta área: 
diferentes tecnologias, softwares para utilização independente ou integrada a sistemas educacionais ou AVAs.

Diferentes algoritmos utilizam variadas informações para diferentes finalidades. Muitos são os relatos de recomendação de grupos e recomendação de objetos de aprendizagem. Isto demonstra que considerar os interesses de alunos e professores pode favorecer a personalização do ensino promovendo resultados significativos (BEHAR, 2019). Um exemplo, é o software que opera junto ao AVA para recomendação de grupos de alunos e utiliza as informações de navegação para obter os atributos a serem processados pelo algoritmo K-Means (MONTEVERDE et al., 2017). O autor aponta elevação no rendimento dos estudantes nas turmas em que a recomendação foi aplicada.

A recomendação de recursos didáticos também é recorrente em publicações sobre o tema. $\mathrm{O}$ armazenamento e catalogação destes recursos permite a busca e reutilização (FABRE; TAMUSIUNAS; TAROUCO, 2003) segundo atributos particulares. A partir da disponibilidade de recursos didáticos, sistemas de recomendação podem selecionar os mais adequados (com base em suas características) para os alunos (com base em suas preferências), segundo os temas que precisam ser trabalhados a cada período letivo pelos diferentes grupos de estudantes (CAZELLA et al., 2009).

\section{Sistema de recomendação de grupos e recursos para transdisciplinaridade}

Considerando que práticas transdisciplinares podem demandar envolvimento de toda comunidade acadêmica, um software que se propõem a potencializar tais práticas deve incluir gestão acadêmica, assessoria pedagógica, professores e alunos. Entretanto, optou-se por restringir a versão inicial do sistema a um componente que possa ter impacto positivo para professores e alunos no processo de ensino e de aprendizagem.

\subsection{Plugin de recomendação de grupos e recursos}

No intuito de oferecer à comunidade acadêmica um software viável e que possa contribuir para ampliação da prática da transdisciplinaridade, entendeu-se como mais adequado iniciar pelo módulo de recomendação de grupos e recursos como um plugin para o AVA Moodle. O ambiente foi escolhido por ser amplamente utilizado. Sua comunidade de desenvolvedores segue ativa, produzindo melhorias no ambiente de aprendizagem assim como em softwares complementares como clientes para smartphones e desktop.

O software desenvolvido leva o nome de GREAT RS, acrônimo para variações em inglês e português de Grupos e Recursos para Abordagem de Temas Transversais. A sigla RS é referência para Sistema de Recomendação (em inglês). O plugin será distribuído sob licença livre, pois espera-se que não apenas seja utilizado por terceiros assim como venha a ganhar melhorias na colaboração com outras pessoas ou equipes que atuem no desenvolvimento de ferramentas para educação.

O plugin é do tipo Bloco e, por tratar-se de um software integrado ao Moodle, sua utilização mais indicada é para instituições ou professores que já estão familiarizados com este ambiente. Sua operação mais básica está ilustrada no fluxograma da Figura 1. Cabe destacar que, segundo a concepção do ambiente Moodle, é utilizado o nome "curso" para o contexto que é geralmente associado a uma disciplina ou componente em uma estrutura curricular tradicional. Assim, sempre que, por opção, for utilizado o nome curso associado ao AVA, estar-se-á fazendo referência à disciplina. 


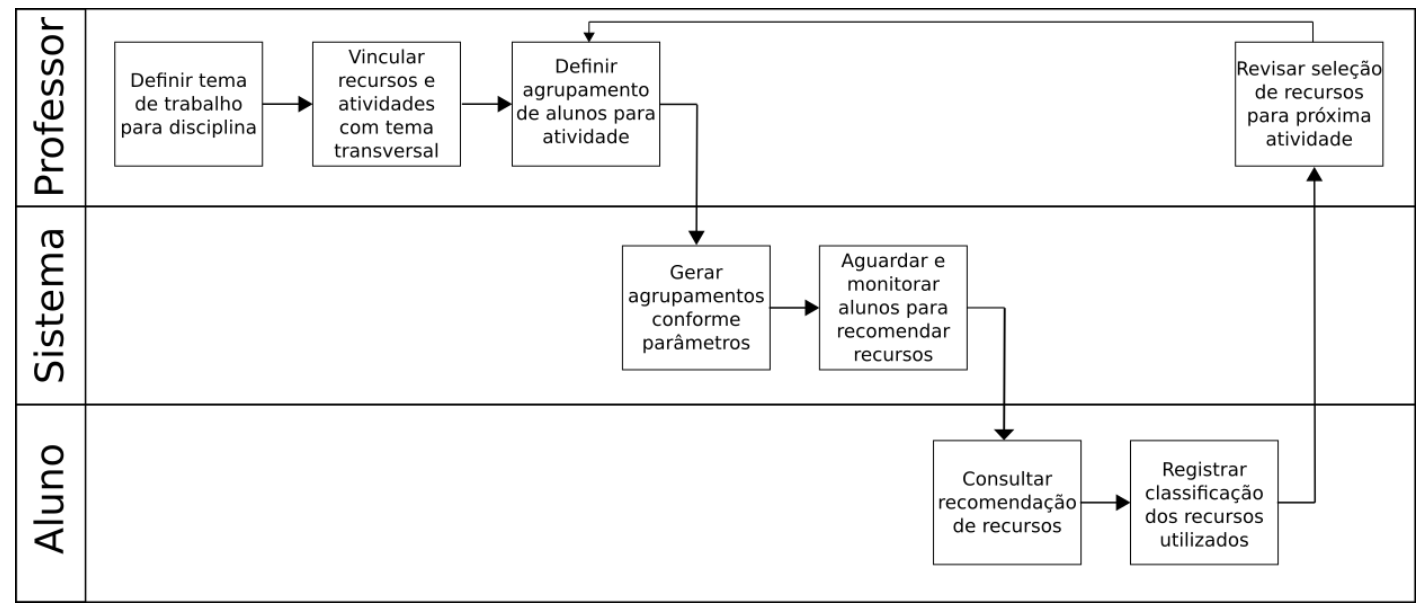

Figura 1. Fluxograma simplificado do funcionamento do sistema

A recomendação de grupos de alunos e recursos didáticos estão fortemente associadas. Grupos são gerados com vistas a trabalhos colaborativos para cumprimento de atividades. Os recursos são recomendados segundo as opções disponíveis e vinculadas em cada seção do conteúdo da disciplina.

\subsection{Navegação e configuração}

O plugin conta com quatro interfaces associadas às funcionalidades principais, além da própria interface do bloco GREAT RS e da sua configuração em cada disciplina. As funcionalidades disponíveis são:

- Vincular recursos e atividades com temas - Permite determinar quais temas transversais estão vinculados a quais recursos ou atividades;

- Definir agrupamentos de alunos - Permite gerar grupos de alunos para diferentes atividades segundo critérios selecionados;

- Ver ranking de recursos - Permite acompanhar ofeedback dos alunos em relação aos recursos avaliados;

- Visualizar recomendações - Útil para revisar os agrupamentos gerados e observar o rendimento dos grupos após encerrada a atividade vinculada.

As figuras a seguir apresentam, respectivamente, as opções de navegação do bloco (Figura 2) e a configuração do mesmo quanto aos temas disponíveis para uso e a habilitação ou não dos componentes de recomendação (Figura 3).

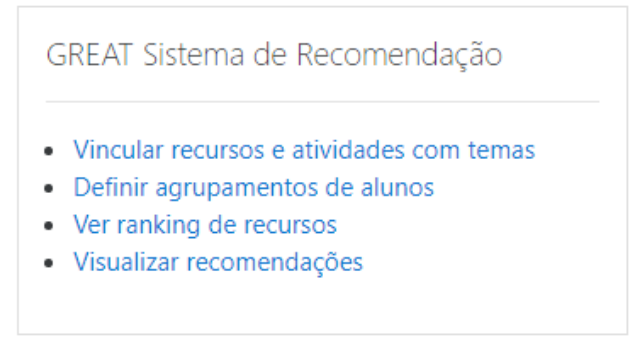

Figura 2. Opções do bloco GREAT RS 


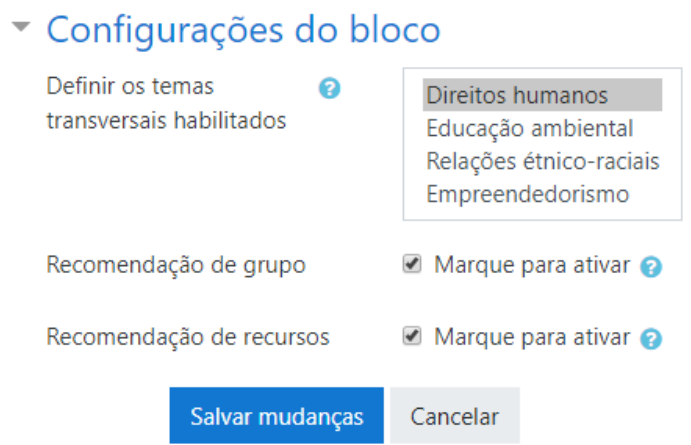

Figura 3. Opções do bloco GREAT RS

\subsection{Recomendação de grupos de alunos}

A recomendação de grupos é tida como recurso central do sistema. Utiliza o algoritmo K-means e é fundamental para determinação dos agrupamentos nas atividades do Moodle, assim como para obtenção da similaridade dos alunos para filtragens de recomendação dos recursos selecionados para tais atividades.

O algoritmo K-means é uma implementação de inteligência artificial do tipo aprendizagem não supervisionada e é amplamente utilizado para problemas de agrupamento e também para recomendação. Tem a função de reconhecer alunos similares considerando determinados atributos. Por este motivo, foi escolhido para ser a primeira opção de algoritmo para recomendações do plugin. Considera-se a adição de outras técnicas de recomendação no futuro, assim como contribuições podem surgir uma vez que o software será oferecido com licença livre.

$\mathrm{Na}$ versão inicial do sistema, existem dois conjuntos de atributos para determinação dos agrupamentos. O primeiro, procura estabelecer o conhecimento prévio dos alunos sobre os temas habilitados para disciplina e o segundo, o perfil de comportamento do aluno na utilização do ambiente.

- Conhecimento prévio no tema - Utiliza a média da pontuação das atividades vinculadas ao tema de referência ao longo das disciplinas que o aluno cursou para apurar o conhecimento. Associado a isto, a contagem destas atividades efetivamente realizadas estabelece o atributo de experiência. O algoritmo utiliza conhecimento e experiência para realizar os agrupamentos;

- Perfil de comportamento - Utiliza um conjunto de indicadores da navegação dos alunos no ambiente Moodle. Os indicadores incluem número de logins, acessos e postagens de tarefas, leituras e postagens em fóruns. O algoritmo utiliza essa medida de navegação para realizar os agrupamentos.

Os acessos no ambiente também são utilizados em outros trabalhos. Exemplos são a utilização de trilhas de aprendizagem (MONTEVERDE et al., 2017) estabelecidas pelo sequenciamento de navegação e de engajamento do aluno (YATHONGCHAI et al., 2013) considerando quantidade e tipos de acessos ao longo do curso.

As figuras a seguir apresentam os parâmetros para geração dos agrupamentos (Figura 4) e o resultado da recomendação, apresentando a lista de alunos com identificação de separadores na listagem que estabelecem os grupos gerados (Figura 5). 


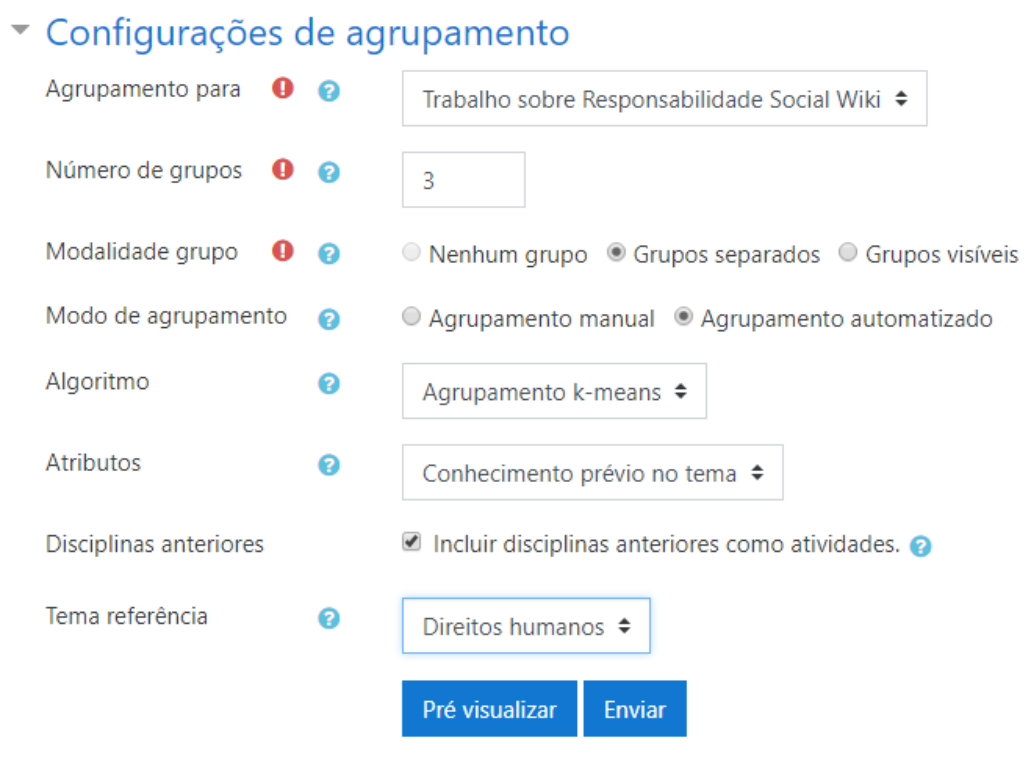

Este formulário contém campos obrigatórios marcados com $\mathbf{Q}$

Figura 4. Parâmetros da recomendação de grupos de alunos

Tanto na Figura 4 quanto na Figura 5, podem ser observados, respectivamente, o parâmetro e o resultado da indicação de inclusão do rendimento em atividades vinculadas ao tema de referência em disciplinas anteriores, registradas e preservadas no ambiente. Ainda na Figura 5, cabe destacar, as colunas identificadas por Disc B 2017-2, Disc E 2018-1 e Disc F 2018-2 trazem a média das atividades vinculadas ao tema de referência em disciplinas anteriores.

\subsection{Recomendação de recursos}

No sistema desenvolvido, a recomendação de recursos tem o objetivo de subsidiar os trabalhos para o cumprimento de determinada atividade. Nesta primeira versão do sistema, o agrupamento de alunos segundo perfil de comportamento é utilizado para determinar o nível de engajamento dos estudantes. A filtragem empregada considera que estudantes com mesmo nível de engajamento podem ter interesses semelhantes quanto ao tipo de material. Assim, recursos melhores qualificados entre alunos dentro de um mesmo grupo de engajamento, serão apresentadas aos demais estudantes.

A vinculação dos recursos direcionados para determinadas atividades é realizada implicitamente pela organização do conteúdo da disciplina no Moodle segundo a divisão por seções (tópicos ou semanas). Isto é, recursos em determinada seção são elegíveis para atividades na mesma seção. Contudo, a recomendação propriamente dita ocorre de forma automática e em momento relativo. Uma configuração de agendamento do plugin executa uma verificação de oportunidade de recomendação.

O padrão de execução estabelecido determina que a recomendação ocorra em uma fração do tempo decorrido entre a recomendação dos grupos para a atividade e o prazo limite para entrega da mesma. Para versão inicial a fração empregada é de $50 \%$. Isso significa, por exemplo, que para um agrupamento gerado 4 semanas antes do prazo de entrega da atividade, a recomendação de recursos irá ocorrer 2 semanas antes deste prazo. A recomendação poderá ser antecipada caso: 


CINTED-UFRGS Novas Tecnologias na Educação

\section{Disc H 2019-1 - Estudantes}

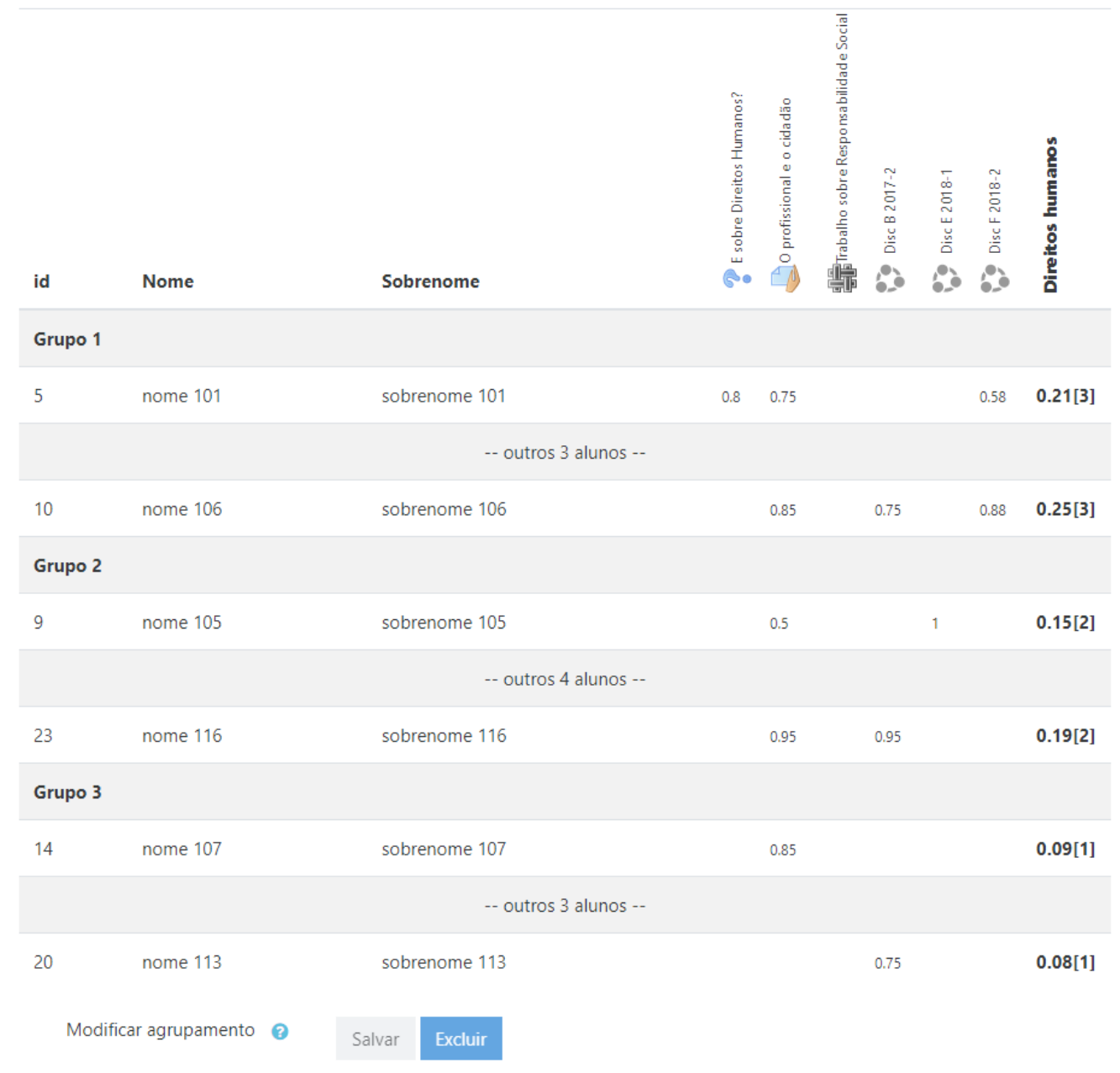

Figura 5. Resultado da recomendação de grupos de alunos

- $50 \%$ dos alunos realizarem acesso aos recursos;

- $30 \%$ dos alunos realizarem a entrega da atividade (quando não houver grupo);

- $15 \%$ dos grupos realizarem a entrega da atividade;

- $20 \%$ dos alunos registrarem avaliação de recursos;

Cabe destacar que um problema comum na recomendação de recursos didáticos - o de não haver nenhuma avaliação útil para recomendação - será minimizado, pois o professor ou tutor poderá realizar a determinação de relevância e qualidade dos recursos trazendo algum subsídio para recomendação.

Ainda assim, a qualificação dos recursos utilizados pelos alunos é parte importante do sistema. Para tanto, os alunos utilizam interface específica para registrar qual a relevância do recurso em relação à atividade e qual a qualidade do mesmo. Para ambos os indicadores é utilizada a escala Likert.

\section{Outras características e informações sobre o sistema}

A proposta do plugin é oferecer benefício em primeira mão essencialmente para professores, mas também para alunos. Docentes com experiência no uso do Moodle podem vincular facilmente recursos e atividades aos temas relacionados com a disciplina. 
Quando não houver atividade anterior para subsidiar a apuração de conhecimento ou experiência prévia, o professor poderá utilizar a atividade "Escolha" para realizar um levantamento junto aos alunos da turma quanto à sua familiaridade com um tema transversal específico. O sistema irá tratar adequadamente opções sequenciais numéricas de qualquer escala, como índice de aproveitamento similar a uma atividade no Moodle. Além disso, outros temas transversais podem ser habilitados no sistema, permitindo que professores e alunos de disciplinas relacionadas à temas variados se beneficiem da recomendação de grupos ou da recomendação de recursos.

A utilização de recomendações combinadas, pelo cruzamento de grupos formados segundo comportamento (engajamento) com grupos formados segundo conhecimento e experiência prévia, procura estabelecer grupos de alunos com similaridade de hábitos de acesso e complementaridade de experiência quanto aos temas, proporcionando sintonia na forma de trabalho e colaboração entre os pares na realização da atividade.

As simulações ocorreram segundo o uso de recursos básicos de uma disciplina Moodle associado à utilização das funcionalidades centrais do plugin. Mudanças nos indicadores de acessos simulados dos alunos produziram a mudança esperada no agrupamento por perfil de comportamento. Mudanças nas notas dos alunos em atividades anteriores da disciplina ou em componentes curriculares cursados previamente causaram mudança apropriada no agrupamento por conhecimento prévio no tema.

\section{Considerações Finais}

Diferentes estudos demonstram que sistemas de recomendação educacional podem incrementar os resultados em processos educativos. O sistema apresentado neste relato oferece recursos que podem ajudar em atividades colaborativas por meio de grupos selecionados segundo similaridade de perfil e níveis de conhecimento prévio construído na trajetória do estudante em disciplinas anteriores apoiando iniciativas transdisciplinares.

A seleção de grupos que possam trabalhar melhor e com maior aproveitamento auxilia o professor encorajando para práticas inter e transdisciplinares. A recomendação de recursos com indicação de relevância e qualidade auxilia os estudantes na seleção dos materiais de estudo e reduz o desperdício de tempo na busca por fontes apropriadas.

Em breve o plugin será disponibilizado no repositório de plugins do Moodle. Além da busca pela aplicação e validação prática do software no escopo da presente pesquisa, a disponibilização da ferramenta para toda comunidade acadêmica favorece a troca de experiências e aperfeiçoamento do sistema.

\section{Referências}

AGUIAR, J. J. B. et al. Um Mapeamento Sistemático sobre Iniciativas Brasileiras em Sistemas de Recomendação Educacionais. In: Anais do SBIE. [s.n.], 2014. v. 25, p. 1123. Disponível em: <http://br-ie.org/pub/index.php/sbie/article/view/3058>.

ARAÚJO, U. F. Temas transversais, pedagogia de projetos e mudanças na educação. São Paulo: Summus, 2014. 120 p. ISBN 978-85-323-0958-7.

BEHAR, P. A. Recomendação Pedagógica em Educação a Distância. Porto Alegre: Penso, 2019. 194 p. 
BERNARDES, M. B. J.; PRIETO, É. C. Educação Ambiental: disciplina versus tema transversal. REMEA, v. 24, p. 173-185, 2010. Disponível em: $<$ https://www.seer.furg.br/remea/article/view/3891>.

BRASIL. Parecer CNE/CES n $n^{\circ} 776 / 1997$, de 3 de dezembro de 1997. Orientação para as diretrizes curriculares dos Cursos de Graduação. 1997. Disponível em: $<$ http://portal.mec.gov.br/cne/arquivos/pdf/CES0776.pdf >.

BRASIL. Instrumento de Avaliação de Cursos de Graduação - presencial e à distância. 2015. $50 \mathrm{p}$.

BUSQUETS, M. D. et al. Temas Transversais em Educação: Bases para uma formação integral. 6. ed. Madri: Editora Ática, 2003. 198 p.

CAMPOS, A. d. et al. Mapeamento de soluções tecnológicas em sistemas de recomendação educacionais em âmbito brasileiro. Informática na educação: teoria \& prática, v. 20, n. 3 set/dez, p. 78-93, feb 2018. ISSN 1982-1654. Disponível em: $<$ https://seer.ufrgs.br/InfEducTeoriaPratica/article/view/79624>.

CAZELLA, S. C. et al. Recomendação de Objetos de Aprendizagem Empregando Filtragem Colaborativa e Competências. In: Anais do Simpósio Brasileiro de Informática na Educação. Florianópolis, SC: [s.n.], 2009. v. 1.

DINARDI, A. J.; ARAUJO, S. C. Inserção da temática ambiental nos cursos de graduação da Universidade Federal do Pampa-Uruguaiana/RS. Debates em Educação, Universidade Federal de Alagoas (UFAL). Centro de Educação. Programa de Pós-Graduação em Educação, v. 9, n. 17, p. 58-78, apr 2017. Disponível em: <http://www.seer.ufal.br/index.php/debateseducacao/article/view/2098>.

FABRE, M.-C. J.; TAMUSIUNAS, F.; TAROUCO, L. M. R. Reusabilidade de objetos educacionais. RENOTE - Revista Novas Tecnologias na Educação, v. 1, n. 1, p. 1-11, feb 2003. Disponível em: <http://seer.ufrgs.br/index.php/renote/article/view/13628>.

MONTEVERDE, I. et al. M-Cluster: Uma ferramenta de Recomendação para Formação de Grupos em Ambientes Virtuais de Aprendizagem. In: Anais do SBIE. Recife, PE: [s.n.], 2017. v. 28, p. 1657-1666. Disponível em: <http: //www.br-ie.org/pub/index.php/sbie/article/view/7697>.

MOZENA, E. R.; OSTERMANN, F. Uma revisão bibliográfica sobre a interdisciplinaridade no ensino das ciências da natureza. Ensaio Pesquisa em Educação em Ciências (Belo Horizonte), v. 16, n. 2, p. 185-206, aug 2014. ISSN impressa ISSN 1415-2150 on-line ISSN 1983-2117. Disponível em: <https://periodicos.ufmg.br/index.php/ensaio/article/view/10149>.

NICOLESCU, B. Manifesto da transdisciplinaridade. São Paulo: [s.n.], 1999. 168 p.

SUANNO, M. V. R. Didática e trabalho docente sob a ótica do pensamento complexo e da transdisciplinaridade. 493 p. Tese (Doutorado) — Universidade Católica de Brasília, 2015.

YATHONGCHAI, C. et al. Learner classification based on learning behavior and performance. In: 2013 IEEE Conference on Open Systems (ICOS). Kuching, Malaysia: [s.n.], 2013. p. 66-70. Disponível em: <https://ieeexplore.ieee.org/document/6735050>. 